Original Research Paper

\title{
Development of an Anthropomorphic Gripping Manipulator: The Analysis of Implementation Methods
}

\author{
${ }^{1}$ Ivan Vladimirovich Krechetov, \\ ${ }^{2}$ Arkady Alekseevich Skvortsov and ${ }^{1}$ Pavel Sergeevich Lavrikov \\ ${ }^{I}$ Office of Scientific Research and Development, \\ Moscow State University of Mechanical Engineering (MAMI), Moscow, Russia \\ ${ }^{2} R U$. Robotics, Moscow, Russia
}

Article history

Received: 08-10-2015

Revised: $12-11-2015$

Accepted: 16-11-2015

Corresponding Author: Ivan Vladimirovich Krechetov Office of Scientific Research and Development, Moscow State University of Mechanical Engineering (MAMI), Moscow, Russia

Email: monblan.pro@yandex.ru

\begin{abstract}
The object of the article is the study of implementation methods for the development of hardware-software engineering solutions in the field of the creation of a universal anthropomorphic gripper. Such technical solutions are intended to enable the adaptation and extensive use of advanced robotic systems in the natural environmental conditions formed by people through equipping them with anthropomorphic universal grippers able to grip, hold and manipulate objects with arbitrary geometric shapes. During the study the anatomical structure of the hand was analyzed. The design and construction of anthropomorphic grippers represented in scientific articles and patents were considered. Also the structure of servomotors and sensor systems were analyzed which provide feedback and adaptability of control. As a result of conducted research trends in the technology of anthropomorphic gripping manipulators were identified. Before the development of a working prototype a comprehensive virtual simulation of a gripper is planned to be conducted. For this purpose Matlab as well as Gazebo, GraspIt and MoveIt included in the Robotic Operation System (ROS) were selected. Using Matlab a mathematical model was created which describe the dynamics and kinematics of the future prototype. For the visualization of the modeling process and the creation of a virtual surround space a mathematical model in Gazebo was developed. Concurrent simulations in these environments allows for the development of algorithms and control software. Motion simulation of the hand in conditions close to real usage allows for the optimization of kinematic and mechanical design.
\end{abstract}

Keywords: Robotics, Anthropomorphic Grip, Anthropomorphic Manipulator, Bionic Arm Prosthesis, Matlab, ROS, GraspIt, MoveIt

\section{Introduction}

\section{Introduce the Problem}

Robotics is a popular and very promising development trend in science and engineering. Both in everyday life and in production (Roboforce, 2015) it is used more and more as different robotic systems including the anthropomorphic are developed. The great advantage of anthropomorphic robots is their ability to work in the "human" environment without additional adaptation. The "human" environment means that it is created by man for himself, to complete tasks which impact on his/her life. For example: Using usual tools and mechanisms to grab and manipulate various objects. In other words the goal is to replace the requirements of man in many fields of activity. One barrier that complicates the widespread use of such robotic systems is the need for an anthropomorphic grab function which could qualitatively replicate the hand. This is especially strongly shown in the tasks associated with the precise handling of small objects and tools with irregular shape where the standard robotic grippers aren't applicable.

A similar problem occurs for developers of bionic upper-limb prostheses where additional increased requirements on weight, power consumption and aesthetic appearance of the structure are imposed. Many scientific 
organizations, commercial companies and even individual researchers and developers are working on their own projects in the development of anthropomorphic grippers and have achieved some success. However, leaping ahead, it is worth noting that most of the projects are exclusively scientific, prototypes are built in a single copy which has a very complicated structure and their commercialization is not obvious or suitable.

The goal of this project is the development of an anthropomorphic gripper which will be able to copy the dynamics and kinematics of the hand with a high degree of accuracy. The field of application for this robotic hand will be industrial robots for replacing people at work, personal robot-assistants and bionic prostheses. At the same time, during development, there is a planned focus on the use of widespread and cheap components and methods to simplify the design to ensure low production costs.

\section{Theoretical Bases and Research Tasks}

The first necessary thing is to consider the biological prototype-a human hand. Figure 1 shows the anatomy and the kinematics scheme of the hand. The diagram presents single-axis joints marked with thick lines and double-axis joints marked with crosses. The dashed line represents a flexible kinematical connection, consisting not of bones but made of the skin and ligaments. In Fig. 1 the following abbreviations are accepted:

- $\quad$ Distal-Middle Joint (DMJ)

- $\quad$ Proximally-Metacarpal Joint (PMTJ)

- $\quad$ Proximally-Middle Joint (PMDJ)

- Intermetacarpal Joint (IJ)

- Carpometacarpal Joint (CJ)

Consider in more detail the kinematic diagram of the hand. The joint PMTJ1 of the thumb has two degrees of freedom, but a wide working range which it has along only one axis. On the second axis deviations do not exceed a few degrees and to simplify the design they can be neglected so we only require a single-axis joint in this knuckle. Similarly in joints PMTJ2, PMTJ3 and IJ3, their relative movement is small and they can also be neglected. The solution is to join these knuckles with a rigid connection:

- IJ4 and IJ5 knuckles have a much longer operating range and can vary depending on a given person's flexibility. Due to this variability there are three options: Each of these knuckles should be independent

- IJ4 should be movable with a rigid connection with IJ5

- IJ4 rigidly connected with IJ3 and IJ5 remains movable

Mankind has independent use of these knuckles. This provides a more reliable means for capturing large round objects, but their implementation in robotic grabbing increases the design complexity and reduces its durability and reliability. The feasibility of using IJ4 and IJ5 is the first subject being analyzed in the next stage of this project-modeling.

Another feature of the hand structure is the drive elements-the muscles are not located directly in the hand but are located in the forearm and connected to the phalanges with flexible tendons. Therefore antagonistic control is implemented. In other words there are two muscles which in succession rotate the phalange to their side.

In the hand itself there are muscles that move the fingers in the plane of the palm and are able to develop a relatively low force, compared with the main flexor. With one exception-the muscle of the thumb which, controls knuckle $\mathrm{CJ} 1$, has very high torque. Arrangement of the drives in the hand or forearm is the second task of the study.

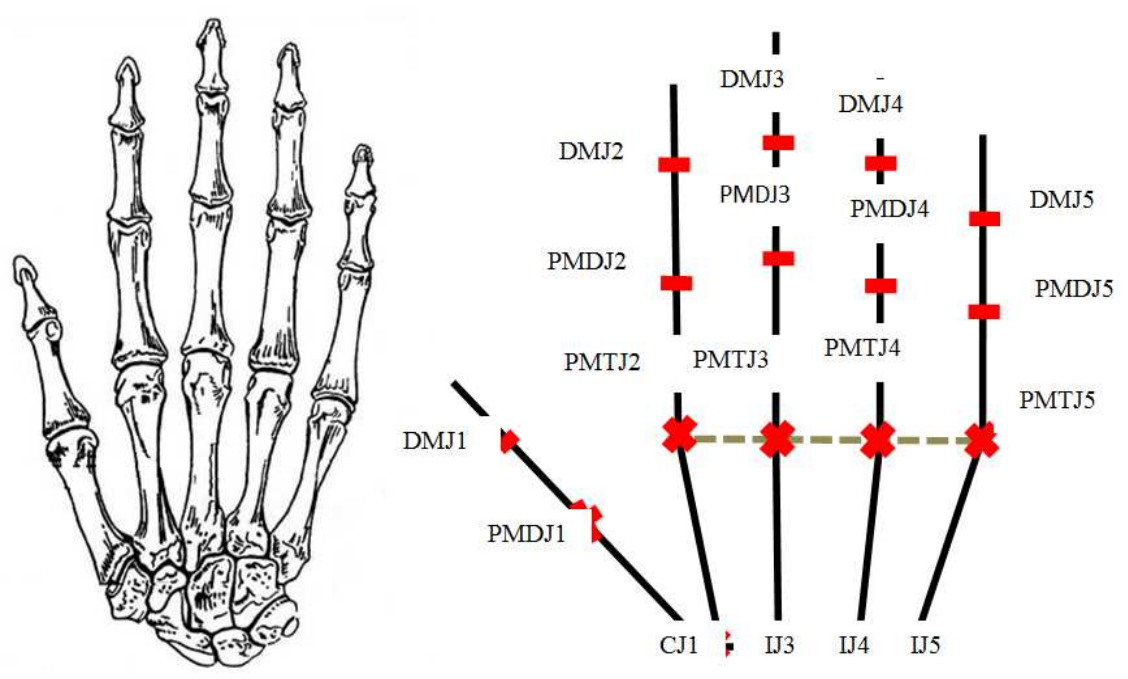

Fig. 1. Kinematic scheme of the hand 
Besides the kinematics arrangement and mechanical design, implementing the skeleton section of the arm requires the mechanical analog of human muscles or an approximation thereof. It is this drive which will rotate the phalanges. Human muscles have amazing properties. They are able to perform precise microscopic movements simultaneously; a prime example would be during surgical operations and especially in neurosurgery and at the same time ensure the continued ability to grab and hold heavy loads, like athletes in weightlifting. Muscles have a very high power-weight ratio. One hand of an average adult male generates forces up to $40-50 \mathrm{~kg}$, an average of 10-12 kg per finger. Thus, a person can strain muscles enough to securely fix fingers as well as completely relax them i.e., to change the stiffness of the knuckle. The third goal of the research is to find a suitable drive method which can meet the requirements of high positioning accuracy and the necessary driving force. Thus, the task of developing an anthropomorphic gripper can be divided into the following subtasks:

- Mechanical design

- Development of the servo drives

- Development of the feedback sensors

During the first stage of the project it is necessary to choose the arrangement solutions for future virtual simulations and verification of the construction performance.

\section{Materials and Methods}

\section{Analysis of the Implementation Methods of the Mechanical Construction}

As mentioned above, there are two possible types of mechanical construction arrangement: With the drives inside the hand and with drives on/in a forearm section. From the point of view of the application versatility of the hand, as part of robotic manipulators, the first type of arrangement is more preferable, i.e., with the drives inside the hand due to the compact design and ease of installation. However, the space constraint inside the hand does not allow us to create a large number of axes and use servo drives capable of generate high forces. One of the first anthropomorphic grippers, which still continues to be the most high-tech and advanced, is the Shadow Dexterous Hand (Shadow Robotics, 2013) from Shadow Robot company (Fig. 2). This grab has 24 independent controllable degrees of freedom (two of them related to the carpal joint) as well as touch, temperature and force sensors. The grab is designed in accordance with the principles of biomimetics and contains a drive which uses artificial pneumatic muscles operated by compressed air supplied through valves with electronic control. In Humans the core muscles are arranged along the forearm to perform flexion and extension of the fingers and the driving force through to the phalanges transmitted through the tendons. Similarly, Shadow Dexterous Hand's muscles are located along the element which simulates the forearm. The transfer of force from the muscles to the joints is carried out through flexible rods simulating the tendons which are laid inside an incompressible flexible braid. Another anthropomorphic gripper which implemented a large number of interesting and promising solutions is the DLR Hand Arm System developed by the German Aerospace Center (Deutsches Zentrum für Luft-und Raumfahrt) (Fig. 2). The paper (Grebenstein et al., 2010) presents the results of research into the structure and kinematics of a human hand as well as the development of the joints, structurally similar to human knuckles: Conventional joint with a single degree of freedom, condyle-like knuckle with two degree of freedom (condyle is the round prominence at the end of a bone serving for attachment of muscles or being a part of a pin-joint) and saddle-like joints with two degrees of freedom. The paper (Stillfried and van der Smagt, 2010) demonstrated that the human hand has 6 knuckles with two degrees of freedom and 12 knuckles with one degree of freedom. Moreover, all one-axis knuckles are condyle-like and the two-axis knuckles are saddle-like. A feature of the condyle-like joint is that it provides a complete rotation around one axis and a small and limited rotation around another axis. This explains the fact that human phalanges may deviate within a small angle around the axis passing through the knuckles and perpendicular to the plane of the hand. The authors of the article decided to implement joints similar to those found in human knuckles.

Both considered grippers, DLR Hand Arm System and Shadow Dexterous Hand, configured similar to human hands, have drives arranged in the forearm. However, there are alternative structural possibilities where the drive is located directly in the hand.

In the German Aerospace Center an anthropomorphic drive located in the hand has also been designed. It is called the DLR Hand II (Fig. 3). A feature of this gripper is that each finger is a separate complete construction (Butterfaß et al., 2001) and has four degrees of freedom, three of which are independent and controlled by individual drives and one dependent DMJ joint which is connected with a PMDJ joint. The structure of each finger includes angular position sensors, force sensors and six-axis force and torque sensors located at the tip of the distal phalanx. This sensor allows the user to monitor touch and determine the direction of occurring force. Each finger is able to generate a force of $30 \mathrm{~N}$ at the end of the distal phalanx. 

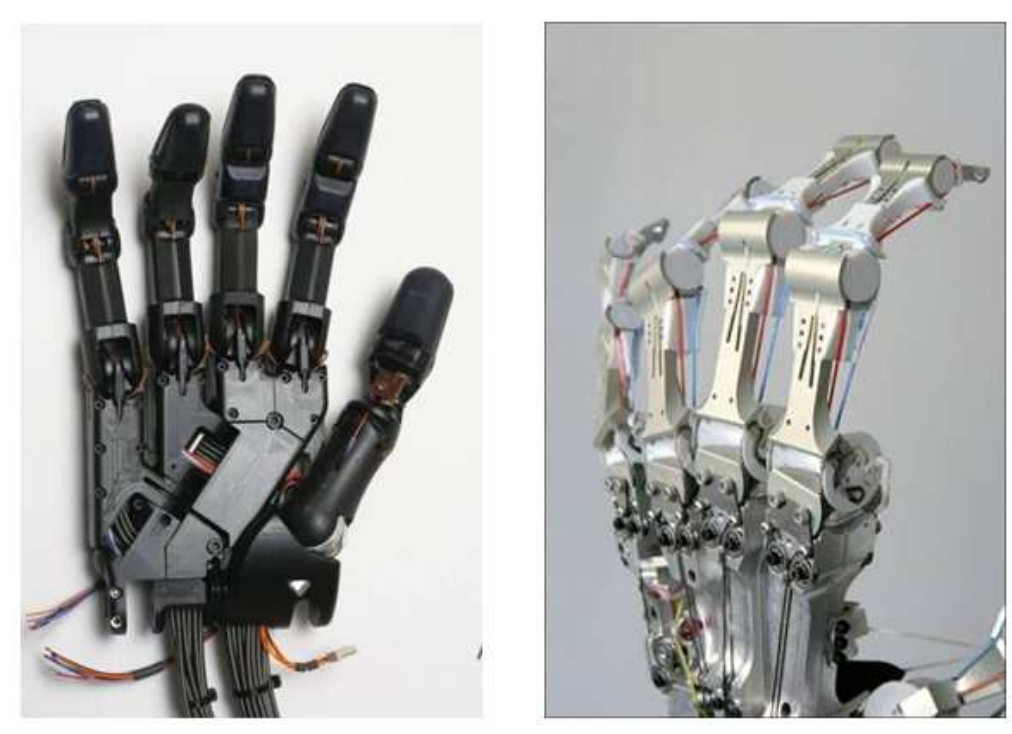

Fig. 2. Shadow dexterous hand and DLR hand arm system
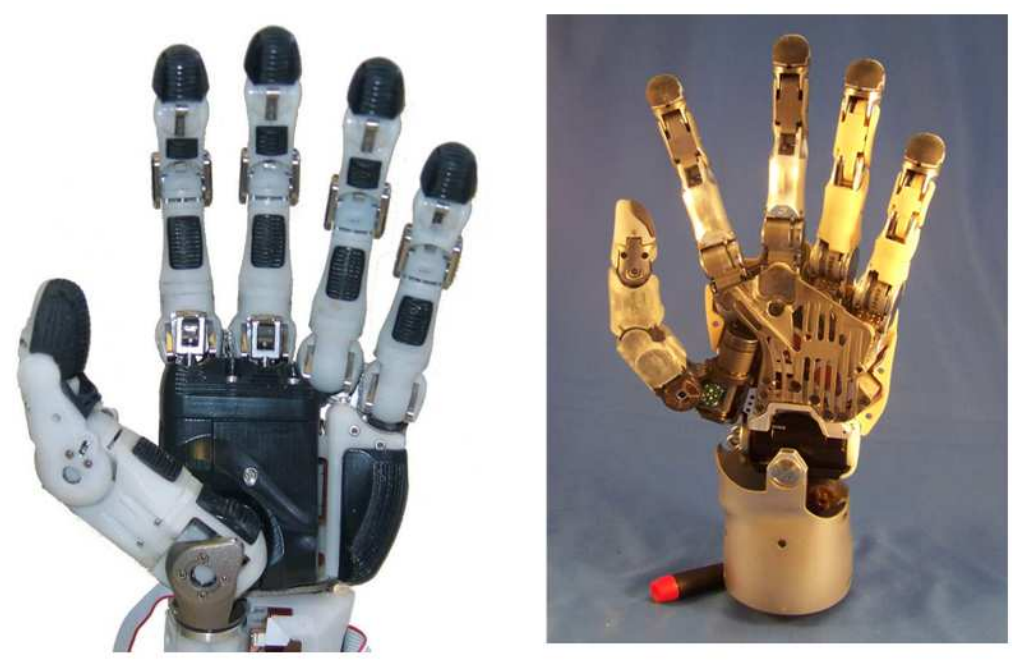

Fig. 3. External view of the DLR hand II and kinea design hand

Based on the DLR Hand II design the company Elumotion developed the ELU2-HAND gripper. The mass of the original structure was heavily reduced (Elu2-Hand, 2010) due to the extensive use of engineering plastic instead of metal so the overall weight was reduced from 1800 to $900 \mathrm{~g}$. This grabber is able to generate a force, at the end of the distal phalanx, equal to only $4.7 \mathrm{~N}$. The construction of this gripper was brought into serial production and is currently part of a larger project where the full torso of an anthropomorphic robot is developing. Figure 3 shows an exterior view of the ELU2-HAND gripper.

A further anthropomorphic gripper, where the drives are located directly in the hand, has been implemented by Kinea Design (Fig. 3). This task is included in the project supported by Defense Advanced Research Projects Agency
(DARPA). The project was launched in order to develop bionic prostheses for people with disabilities. The gripper is complete with seven degrees of freedom.

There are some modifications to anthropomorphic grippers designed to be used as bionic prostheses. The most famous and innovative of them are i-Limb (i-Limb ultra, 2013) and (beBionic, 2013). They have similar mechanical design featuring 6 controlled degrees of freedom and a control system with programmed patterns for gripping and holding objects. A special feature of these robots is the simplified construction and leak-tight design.

\section{Analysis of the Implementation Methods of the Drive System}

A feature of the DLR Hand Arm System gripper, which distinguishes it from other robot design types, is 
the implementation of drives with controlled stiffness/rigidity. Implementation of controlled stiffness and flexible rods causes an antagonistic drive scheme as shown in Fig. 4. The letter «K» denotes the stiffness of the elastic element.

According to this scheme the joint is controlled using two drives. One of them rotates the joint clockwise and the second counterclockwise. In the paper (Grebenstein et al., 2011) the authors describe electrical servo drives specially developed for the DLR Hand Arm System gripper. A three-phase brushless electromotor with a peak power of about $180 \mathrm{~W}$ and a planetary gearbox is located at the heart of the servo drive. Motor control is implemented by a programmable logic integrated circuit (hereinafter FPGA), Xilinx Spartan 3e XC3S500EP132 and performed at a frequency of $100 \mathrm{kHz}$. Each servo drive is designed as a complete individual module to allow for the quick replacement of defective drives.

All joints of the grabber are controlled with an antagonistic scheme, i.e., two drives deflect the joint, each to its own side and the resulting movement of the joints is the sum of the displacement of both drives. Establishing the additional elastic elements allows for the implementation of joint stiffness control. Figure 5 shows a diagram of the elastic element. It is a springloaded lever where one end is pivotally attached to the housing. The tension spring is attached to a second end.
Two pulleys are attached onto the lever itself through which the flexible rod from the drive to the executive element is fixed. During its rotation the flexible rod is wound on the spool mounted on the output shaft of the drive and moves the corresponding element. When an external load or barrier is encountered the flexible rod is tensioned which deflects a spring-loaded lever. Measuring the lever's angular position permits the force in the joint to be monitored without additional specialized sensors. Thus, when an external shock load appears it is not passed onto the drive and gear wheels but absorbed due to the absorption properties of the elastic element thereby providing the joints high resistance to external influences.

In the paper (Semini, 2010) the authors present a comparison of drives according to working principle. Hydraulic drives are not useful in a hand due to the large volume, weight and complexity of the hydraulic cylinders, valves and the oil pump.

\section{Analysis of the Implementation Methods of the Tactile Sensors}

The system of tactile sensors is designed to provide a feedback function in the tasks where the reliable hold of an object in the manipulator is necessary. The basic functions of the sensors are: Contact identification of the gripper with an object, definition of the force generated on the captured object, definition of the normal vector at the point of contact.

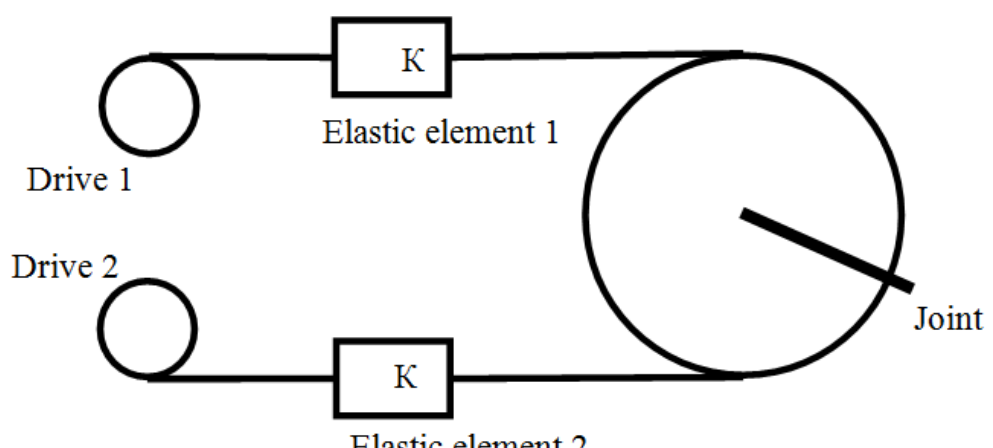

Elastic element 2

Fig. 4. Antagonistic scheme of the drive

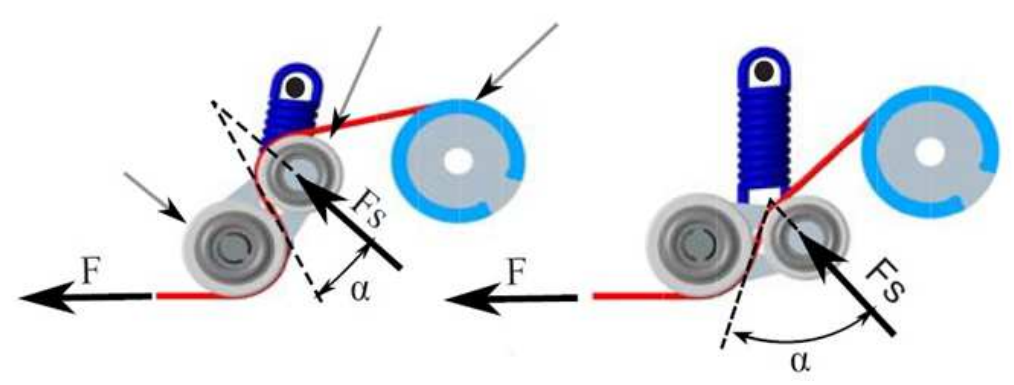

Fig. 5. Diagram of the elastic element 
The tactile sensors are designed using a micromechanical device that converts mechanical energy into electrical. To achieve a tactile stimulation comparable with human sense capability, it is necessary to provide a resolution in the sensor of not less than 1 $\mathrm{mm}$. According to the working principle tactile sensors can be divided into the following groups:

- Resistive

- Capacitive

- Transistored (POSFET)

- Piezoelectric

The working principle of the resistive sensors is based on circuit closing under the influence of applied force. The deformable body has a conductive layer which comes into contact with a terminal pad (electrodes) made in the form of a periodically repeating structure (meander).

The objects of study for such sensors are the material parameters of the resistive layer and deformable body. The paper (Schurmann et al., 2011) presented a $16 \times 16$ array of sensors with a total size of $80 \times 80 \mathrm{~mm}$. Typically, the deformable body material is a siliconebased elastomer or polyurethane. The conductivity of the elastomer depends on the deformation that is provided by the graphite particles that make up the material.

The electrical circuit consists of a 16-channel analogto-digital converter and for each input through the multiplexer an array of $4 \times 4$ sensors is connected. It is shown, experimentally, that the electrodes shape and the distance between them has an influence on the sensors sensitivity. Research into foamed elastomer materials has showed their ability to withstand pressure up to $30 \mathrm{kPa}$. The main drawback of such sensors is their size and, consequently, low resolution in the range of 3.5 to $5.0 \mathrm{~mm}$. Elastomer parameters impose a limit on the range of measured pressure values. The paper (Zhang et al., 2013) presented a constructive solution that makes it possible to measure not only the normal component of applied force but also tangential. Measuring the last parameter can significantly improve the quality of the grab control providing slip monitoring of a held object.

In the project related to the development of artificial skin (Schmitz et al., 2011) it was noted that the sensory system is a series of interconnected individual sensor modules. Each module is made in the shape of a triangle and consists of 12 micro capacitive sensors. The triangular shape of the sensor provides coverage for large objects, especially with complicated shapes. The data from the sensors is transmitted to the microcontroller which can process up to 16 units.

The paper (Maiolino et al., 2013) provided an updated design of capacitive sensors consisting of a 4layer flexible printed circuit board. The new design has a low hysteresis, high sensitivity and increased resolution. The authors (Dahiya et al., 2007), (Dahiya and Valle, 2013) developed a mathematical model of the sensor made by sputtering a piezoelectric polymer to the gate of the FET, with the structure of the tactile sensor configured as an array of sensitive $5 \times 5$ cells. The factors affecting the sensitivity and linearity of the sensor parameters are considered. The influence of the coating material is researched and efficiency due to the fluorine plastic (polyvinylidene fluoride) as the substrate material is shown. Each sensor element of the array is made up of a piezoelectric polymer film directly covering the gate area of the FET that provides a significant reduction in sensor size, electrical parasitic interferences and improves signal to noise ratio. The paper (Dahiya et al., 2010) presented a tactile sensor made as a sensor on a chip. The sensor chip consists of a $4 \times 4$ array of sensing elements and 16-channel output consisting of two multiplexers and amplifier cascades. The high degree of integration (step sensing element of $1 \mathrm{~mm}$ ) and a total size for the sensor of $8 \times 10 \mathrm{~mm}$ allows the detector to be used as a tactile sensor for finger phalanges of anthropomorphic manipulators. Tactile sensors on a silicon wafer constructed as a chip has a significant advantage over the sensors made of thin-film technology, allowing for the future implementation of compact solutions with integrated signal processing units within a small structural element.

\section{Virtual Simulation}

To check the system and technical solutions during the next phase of work on the project a comprehensive virtual simulation is planned. The most common and multifunctional mathematical modeling environment is Matlab with Simulink, with Sim Mechanics packs included in it. This environment enables simultaneous mathematical modeling of the mechanical structure as well as control algorithms. However, Matlab has a significant limitation-the lack of a full-featured engine for physical modeling of mechanical components. To eliminate this drawback a connection is planned to the Gazebo module included to the Robotic Operating System (ROS). The operating system has open-source software and is used in a variety of household and industrial robots as it allows the virtual models of the control systems to construct real robots. The GraspIt and MoveIt packs included in the ROS are particularly interesting. The first package is designed for the hand simulation, patterning capture subjects and evaluating their quality. The second package is designed for planning trajectories of hand motion and motion manipulation. Multiple uses of Matlab, Gazebo, GraspIt and MoveIt allow one to perform comprehensive simulations of all systems, control algorithms and software included in the system. 


\section{Results}

In consequence of existing structures analysis, it was decided to take as a starting point, the configuration with servo drives located in the forearm because with such a configuration it is possible to realize a large number of independent degrees of freedom. Moreover, it is possible to use more powerful and larger brushless motors ensuring higher degrees of force. At the same time, large motors with high power ratings have better heat dissipation. There will be much less heat produced, especially in static mode, when the motor generates maximum power. The ability to work without overheating is very relevant for a robot hand as the forearm is expected to contain a very high density of components. Due to this fact the organization of active cooling with fans and massive radiators will be difficult. To achieve the research goals a simplified solid model of a hand with kinematics similar to a human has been developed. Based on this three-dimensional model the virtual mathematical model for complex modeling both in Matlab and ROS was created. In Fig. 6 is a mathematical model of the hand in Matlab Simulink.

Visualization of virtual models in Matlab Simulink and GraspIt is presented in Fig. 7. Conducted an analysis of efficiency use of electrical motors and pneumatic muscles, the result is shown in Table 1.

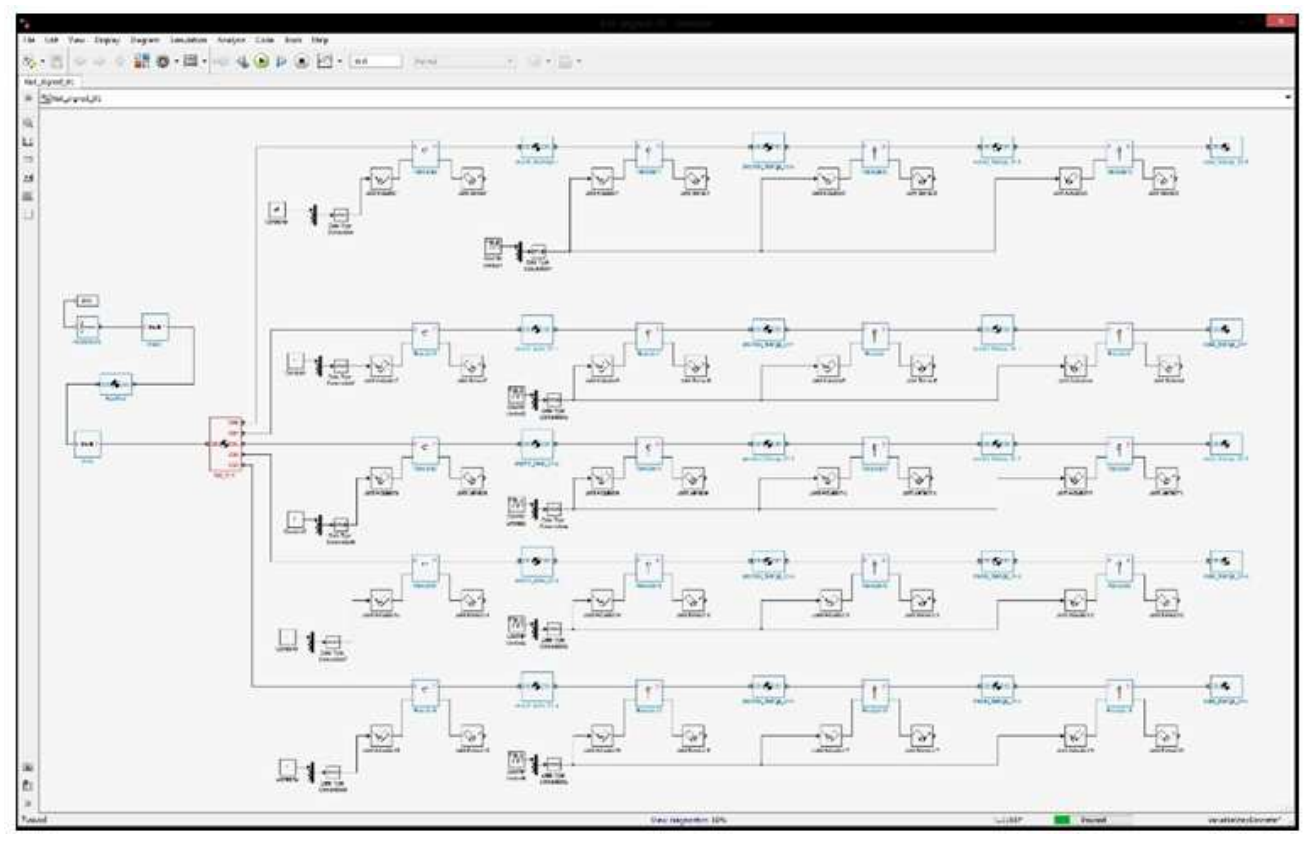

Fig. 6. Mathematical model in Matlab Simulink
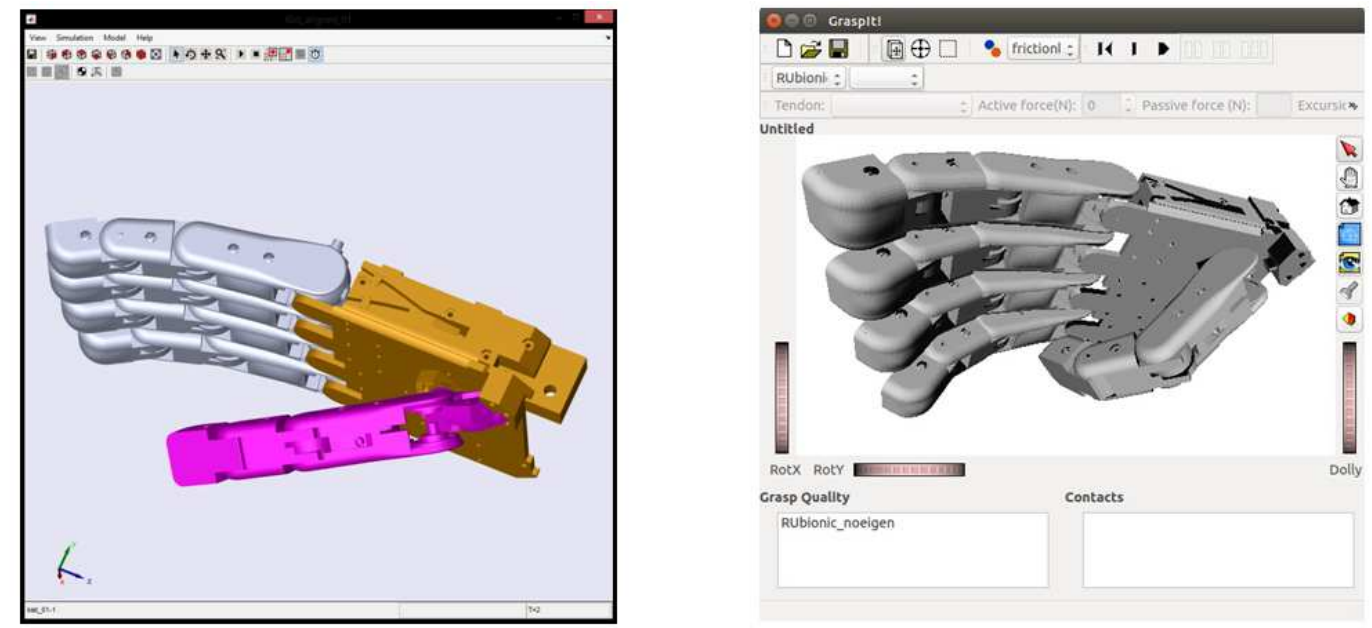

Fig. 7. Visualization in Matlab Simulink and GraspIt 
Table 1. Drives parameters

\begin{tabular}{lll}
\hline Feature & Electrical drive & Pneumatic drive \\
\hline & Electrical drives, & Compressor, air filters, \\
System components & software, gearbox, heatsinks & pneumatic drives, valves. \\
Efficiency coefficient & More than 90\% & Less than 30\% \\
Force to weight ratio & Average & Acceptable \\
Temperature sensitivity & Low & Very high \\
Cost & Low & Low \\
Complexity of the control algorithm & Low & High \\
\hline
\end{tabular}

The model in the Matlab Simulink environment consists of blocks that perform specific functions. The developed model blocks can be divided into the following groups:

- Blocks of three-dimensional solid models of finger phalanges and palm including mass properties and the moments of inertia matrix for the each coordinate axes

- Blocks which are imitating joint hinges. The transmission elements from servo drives and feedback potentiometers are connected to these blocks. In this model, blocks of virtual servo drives control the hinges, which in turn are mathematical models of a real servo drive prototype planned to be used in the developed hand

- Blocks of input and output ports. Through these blocks the information from the ROS Gazebo environment can be transmitted and received. Inputoutput blocks can use any hardware data interface, including USB and Ethernet. Blocks of mathematical calculations also implement data processing algorithms from feedback sensors, computing forward and inverse kinematics and dynamics, as well as servo drives control

The developed mathematical model has the following operation algorithm:

- When the simulation process is started Matlab initializes a three-dimensional model in Gazebo which simulates a real physical prototype of the hand

- The data received from the feedback sensors is transmitted from Gazebo to Matlab. The data includes: The position of the joints, external and drive force influences on the hand as well as information from the tactile sensors

- Blocks of mathematical calculations in Matlab calculate forward and inverse kinematics and the dynamics of the hand's state as described by the feedback sensors

- The calculated data is transmitted to the Gazebo environment which visualizes the calculated state of the hand

- The data transmitted via an Ethernet interface
At the same time, Gazebo allows for the creation of any virtual environment model, such as a manipulator (hand model), as well as various objects and tools for capturing, holding and manipulation.

\section{Discussion}

During the study of materials in the structures of the hand, the authors of this article also looked at manipulators, mobile robots and grippers in general. In many of the analyzed materials it is specified that bionics and biomimetics are becoming more popular among developers of robotic devices and systems. This is confirmed by the increasing number of patents and articles concerning various constructions.

Developed structure in the current stage of the project shows a high degree of similarity between the kinematic scheme and the human hand. A virtual model of the hand will allow for a comprehensive simulation of all systems and a visualization of the results. In the next phase of work, during the modeling process, it is planned that the following tasks are solved: Determine the feasibility of using moving joints IJ4 and IJ5, evaluate the kinematic model, determine the parameters of necessary servo drives. The simulation results allows for final technical solutions and to begin designing a prototype for construction and future manufacturing. At the same time, it is possible to choose the servo drives with the most compatible characteristics or to define the requirements for an individual drive type.

\section{Conclusion}

The GraspIt package (part of ROS) allows for the evaluation of the metrics of capture and to show how reliable it is. In other words, can an object can be held if the unit is in active or continuous motion. At the same time the virtual contact pads on the contact surface of the gripper will show how correctly the tactile feedback sensors are placed. Complex modeling will also simplify the process of developing the control system because the ROS permits the creation of software from the virtual models, including embedded computing tools working in real time mode. As mentioned above, the data transmission in Matlab and Gazebo is carried out via an Ethernet interface. Through this interface the model as well as 
the real control system of the hand can be connected. After developing the control algorithms and testing their efficiency it are plans to generate software for an embedded control system for the hand prototype and then connect it to the virtual model created in Matlab. Thus, quick switching between the virtual model and the real prototype becomes possible. It allows for control algorithms to be directly set onto the real physical system. A network interface provides the ability to remotely control prototypes located in different laboratories. Another important issue is the calculation of the construction strength. In the next step, after the data modeling of kinematics are plans to finalize the three-dimensional solid model and analyze its strength characteristics. This study will allow for the selection of suitable construction materials for the manufacture of a prototype and to eliminate any weakened places within the structure which have a high risk of fracture.

Further to this is an important note. Matlab and ROS are very popular among scientists, engineers and developers of robotic systems. A comprehensive freely distributed virtual model will attract attention in the scientific and engineering community and provide an opportunity to use the hand as part of new robotic projects.

Market analysis simultaneously carried out with research into anthropomorphic grippers showed that once all of the technical features of the gripper are realized demand for this product may grow exponentially. Another words, the gripper prototype has a high potential for commercialization.

\section{Acknowledgment}

This research was financially supported by the Ministry of Education and Science of the Russian Federation under the Grant agreement \# 14.577.21.0136 as of "24" November 2014 (Unique reference identifier of the agreement: RFMEFI57714X0136); the grant is provided to perform the applied research on the topic: "Development of a universal gripping device for performing anthropomorphic type contact operations with high accuracy and reliability". Work on the project is carried out at the Moscow State University of Mechanical Engineering (MAMI). We would also like to thank Pavel Lavrikov from the RU. Robotics (www.rurobotics.com) for providing us with models of their robotic hand.

\section{Author's Contributions}

Ivan Vladimirovich Krechetov: Designed the plan of research, designed kinematic scheme of manipulator, researched implementation methods of the mechanical construction and drive system.

Arkady Alekseevich Skvortsov: Organized the study, data collection of the study sample, analysis and writing of the manuscript.
Pavel Sergeevich Lavrikov: Designed virtual robot hand model based on selected kinematic scheme.

\section{Ethics}

The authors have no conflicts of interest in the development and publication of current research.

\section{References}

beBionic, 2013. Technical information. RSLSteper.

Butterfaß, J., M. Grebenstein, H. Liu and G. Hirzinger, 2001. DLR-Hand II: Next generation of a dextrous robot hand. Proceedings of the IEEE International Conference on Robotics and Automation, May 21-26, IEEE Xplore Press, pp: 109-114. DOI: 10.1109/ROBOT.2001.932538

Dahiya, R.S., A. Adami, M. Valle, L. Lorenzelli and G. Metta, 2010. CMOS implementation of POSFET tactile sensing arrays with on chip readout. Proceedings of 4th International Conference on Sensor Technologies and Applications, Jul. 18-25, IEEE Xplore Press, Venice, pp: 80-83.

DOI: 10.1109/SENSORCOMM.2010.19

Dahiya, R.S., M. Valle, G. Metta and L. Lorenzelli, 2007. POSFET based tactile sensor arrays. Proceedings of the 14th IEEE International Conference on Electronics, Circuits and Systems, Dec. 11-14, IEEE Xplore Press, Marrakech, pp: 1075-1078. DOI: $10.1109 /$ ICECS.2007.4511180

Dahiya, R.S. and M. Valle, 2013. Robotic Tactile Sensing. Springer, New York, USA.

Elu2-Hand, 2010. Specification, Elumotion Ltd.

Grebenstein, M., M. Chalon, G. Hirzinger and R. Siegwart, 2010. Antagonistically driven finger design for the anthropomorphic DLR hand arm system. Proceedings of the 10th IEEE-RAS International Conference on Humanoid Robots, Dec. 6-8, IEEE Xplore Press, Nashville, TN, pp: 609-616. DOI: 10.1109/ICHR.2010.5686342

Grebenstein, M., A. Albu-Schaffer, T. Bahls, M. and Chalon et al., 2011. The DLR hand arm system. Proceedings of the IEEE International Conference on Robotics and Automation, May 9-13, IEEE Xplore Press, Shanghai, pp: 3175-3182. DOI: 10.1109/ICRA.2011.5980371

i-Limb ultra, 2013. Specification MA 01094, Touch Bionics.

Maiolino, P., M. Maggiali, G. Cannata, G. Metta and L. Natale, 2013. A flexible and robust large scale capacitive tactile system for robots. Sensors J., IEEE, 13: 3910-3917.

DOI: 10.1109/JSEN.2013.2258149 
Roboforce, 2015. Chinese plant replaces 90 percent of workers with robots.

Schmitz, A., P. Maiolino, M. Maggiali, L. Natale and G. Cannata et al., 2011. Methods and technologies for the implementation of large-scale robot tactile sensors. IEEE Trans. Robot., 27: 389-400. DOI: $10.1109 /$ TRO.2011.2132930

Schurmann, C., R. Koiva, R. Haschke and H. Ritter, 2011. A modular high-speed tactile sensor for human manipulation research. Proceedings of the World Haptics Conference (WHC' 11), pp: 339-344. DOI: 10.1109/WHC.2011.5945509

Semini, C., 2010. HyQ-Design and development of a hydraulically actuated quadruped robot. PD Thesis, University of Genoa, Italy.
Shadow Robotics, 2013. Shadow dexterous hand el series. Technical Specification, Shadow Robotics.

Stillfried, G. and P. van der Smagt, 2010. Movement model of a human hand based on Magnetic Resonance Imaging (MRI). Proceedings of the International Conference on Applied Bionics and Biomechanics, Oct. 14-16, Venice, Italy, pp: 1-5.

Zhang, T., H. Liu, L. Jiang, S. Fan and J. Yang, 2013. Development of a flexible 3-D tactile sensor system for anthropomorphic artificial hand. Sensors J., IEEE, 13: 510-518.

DOI: 10.1109/JSEN.2012.2220345 